\title{
Endoluminal surgical triangulation: overcoming challenges of colonic endoscopic submucosal dissections using a novel flexible endoscopic surgical platform: feasibility study in a porcine model
}

\author{
Michele Diana $\cdot$ Hyunsoo Chung $\cdot$ Keng-Hao Liu $\cdot$ \\ Bernard Dallemagne • Nicolas Demartines • \\ Didier Mutter $\cdot$ Jacques Marescaux
}

Received: 3 May 2013/Accepted: 23 May 2013/Published online: 21 June 2013

(C) Springer Science+Business Media New York 2013

\begin{abstract}
Background Colonic endoscopic submucosal dissection (ESD) is challenging as a result of the limited ability of conventional endoscopic instruments to achieve traction and exposure. The aim of this study was to evaluate the feasibility of colonic ESD in a porcine model using a novel endoscopic surgical platform, the Anubiscope (Karl Storz, Tüttlingen, Germany), equipped with two working channels for surgical instruments with four degrees of freedom offering surgical triangulation.

Methods Nine ESDs were performed by a surgeon without any ESD experience in three swine, at 25, 15, and $10 \mathrm{~cm}$ above the anal verge with the Anubiscope. Sixteen ESDs were performed by an experienced endoscopist in five swine using conventional endoscopic instruments. Major ESD steps included the following for both groups: scoring the area, submucosal injection of glycerol, precut, and submucosal dissection. Outcomes measured were as follows: dissection time and speed, specimen size, en bloc dissection, and complications.
\end{abstract}

Presented at the 21st Congress of the European Association for Endoscopic Surgery, June 19-22, 2013, Vienna, Austria.

Michele Diana and Hyunsoo Chung equally contributed to this study.

M. Diana $(\bowtie) \cdot$ H. Chung · K.-H. Liu · B. Dallemagne .

D. Mutter - J. Marescaux

Department of General, Digestive and Endocrine Surgery,

IRCAD-IHU, University of Strasbourg, 1, Place de l'Hôpital,

67091 Strasbourg, France

e-mail: michele.diana@ircad.fr

M. Diana $\cdot$ N. Demartines

Department of Visceral Surgery, CHUV, University Hospital

of Lausanne, Lausanne, Switzerland
Results No perforations occurred in the Anubis group, while there were eight perforations $(50 \%)$ in the conventional group $(p=0.02)$. Complete and en bloc dissections were achieved in all cases in the Anubis group. Mean dissection time for completed cases was statistically significantly shorter in the Anubis group (32.3 \pm 16.1 vs. $55.87 \pm 7.66 \mathrm{~min} ; p=0.0019)$. Mean specimen size was higher in the conventional group (1321 \pm 230 vs. $\left.927.77 \pm 229.96 \mathrm{~mm}^{2} ; p=0.003\right)$, but mean dissection speed was similar $\left(35.95 \pm 18.93\right.$ vs. $23.98 \pm 5.02 \mathrm{~mm}^{2} /$ min in the Anubis and conventional groups, respectively; $p=0.1$ ).

Conclusions Colonic ESDs were feasible in pig models with the Anubiscope. This surgical endoscopic platform is promising for endoluminal surgical procedures such as ESD, as it is user-friendly, effective, and safe.

Keywords Anubiscope - Colonic ESD - Endoscopic submucosal dissection (ESD) - Surgical endoscopic platform

Endoscopic submucosal dissection (ESD) is an emerging endoluminal option in the treatment of early stage gastrointestinal cancers with minimum risk of lymph node involvement. ESD offers higher en bloc resection and curative rates than conventional diathermic snare endoscopic mucosal resection (EMR) [1] and can treat larger lesions [2]. In Eastern countries, in the hands of epidemiology-driven skilled endoscopists, ESD is now the standard of care for early gastric cancers [3]. However, ESD is considered a challenging and time-consuming procedure, mainly as a result of the lack of proper operative instruments.

The standard configuration for ESD is represented by conventional endoscopes surmounted by a transparent tip 
hood that exerts tension on the submucosal layer and various endoknives allowing dissection while advancing the scope's tip underneath the submucosal layer [4]. Major technical limitations with this setting include traction, exposure of the submucosal dissection plane and the need to frequently change endoscopic devices (e.g., to perform hemostasis or to switch to a different shaped endoknife), leading to increased risk of perforation and bleeding as compared to EMR and to longer operative times with possible patient discomfort [5, 6]. Such limitations are even more evident when dealing with colorectal polyps because anatomical specificities (e.g., presence of flexures and folds), peristalsis, and residual stools, all of which limit operative field views, may add difficulties to the procedure [7]. Recently published large series of colorectal ESDs demonstrated feasibility and safety in the clinical setting [2].

Several devices and techniques have been developed to facilitate ESD, with the aim of replicating the "traction/ countertraction" surgical axiom in the endoscopic environment.

The endoscopic platform R-scope (Olympus, Tokyo) is equipped with two independently movable working channels: one fits a grasping forceps, which can be moved vertically to lift up the lesion, and the other channel can move an electrocautery knife horizontally to dissect the exposed submucosal layer. This technology has been applied in experimental and clinical gastric ESD [8, 9], and a significant improvement has been demonstrated in operative time and a similar perforation rate compared to conventional ESD.

At the Institute for Research against Digestive Cancer (IRCAD-IHU, Strasbourg, France), a novel surgical endoscopic platform, the Anubiscope (Karl Storz, Tüttlingen, Germany), has been recently developed for natural orifice transluminal endoscopic surgery (NOTES) [10] and laparoendoscopic single-site surgery [11]. The flexible shaft of the Anubiscope, $1.8 \mathrm{~cm}$ in diameter, houses two 4.3-mm and one $3.2-\mathrm{mm}$ working channels, and the tip of the platform opens like a clamshell to space the instruments and offers surgical triangulation. Instruments have an articulated tip and allow for five degrees of freedom and are manipulated by two intuitive handles (Fig. 1). This device has been used at our institute to perform a series of experimental hybrid NOTES procedures [12], and it has been successfully used in the clinical setting for transvaginal cholecystectomy [13].

We aimed to take advantage of the surgical triangulation offered by this endoscopic platform in the endoluminal environment to perform colonic ESDs. The Anubiscope toolbox includes various graspers, a hook knife and insulated tip electrocautery, and an endoscopic needle holder. This randomized experimental study was designed to assess the efficacy of the Anubiscope in performing colonic ESD when compared to conventional endoscopic instruments in an acute porcine model.

\section{Materials and methods}

\section{Animals}

Eight large white pigs (weighing 35-40 kg) were included in the study.

The study protocol was approved by the local ethical committee, and animals were managed in accordance with French laws for animal use and care as well as the European Community Council directive no. 86/609/EEC.

Animals were fasted for $24 \mathrm{~h}$ before the procedure with free access to water. Ketamine $(7 \mathrm{~mL})$ and azaperone (3 mL, Stresnil; Janssen-Cilag, Beerse, Belgium) were administered intramuscularly $1 \mathrm{~h}$ before the procedure as premedication. Induction of anesthesia was achieved using intravenous propofol combined with pancuronium $(2 \mathrm{~mL})$.

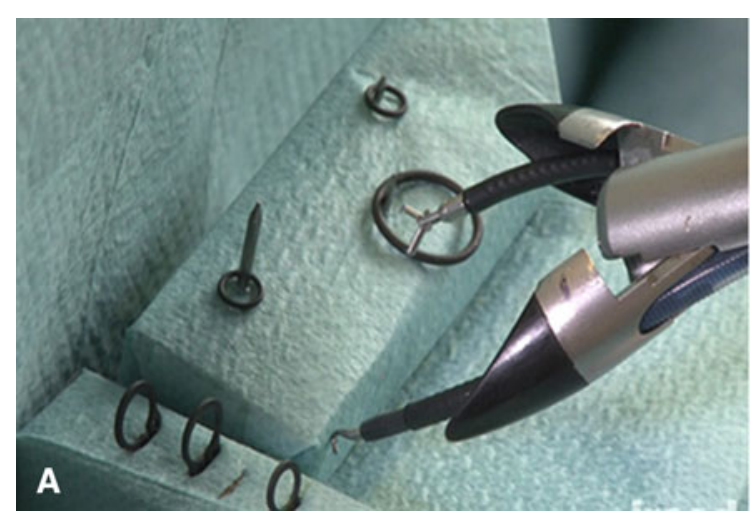

Fig. 1 Handheld mechanical Anubiscope. Anubiscope (Karl Storz, Tüttlingen, Germany) is a prototype of flexible endoscopic surgical platform, equipped with two $4.3-\mathrm{mm}$ and one $3.2-\mathrm{mm}$ working channels as well as a tip that opens like a clamshell to space the

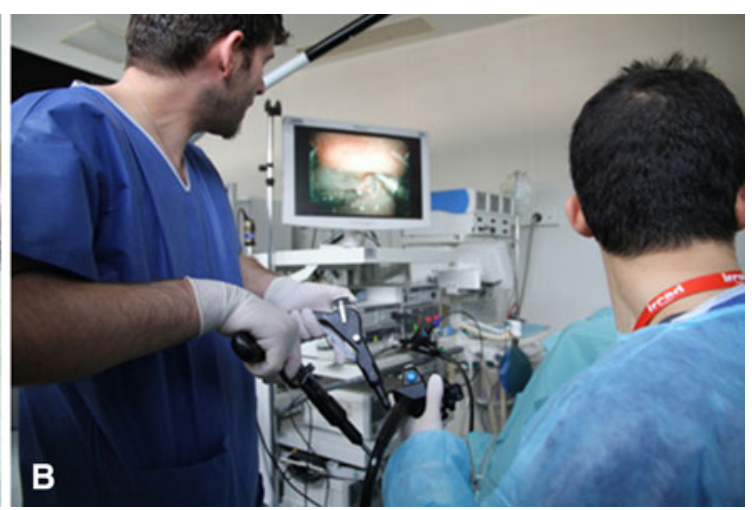

instruments and offer surgical triangulation (A). Instruments have an articulated tip and allow for four degrees of freedom, and are manipulated by two intuitive handles (B) 
Anesthesia was maintained with $2 \%$ isoflurane after endotracheal intubation of the animal lying supine. Rectal cleansing was performed until the effluent turned clear.

All pigs were humanely killed immediately after the procedures by intravenous injection of a lethal dose of potassium chloride.

Pigs were divided into two groups: colonic ESDs with the Anubiscope $(n=3)$ and with conventional endoscopic instruments $(n=3)$. The per-protocol sample size included six pigs, but two additional animals were required in the conventional endoscopic group.

\section{Procedures}

The original protocol included a cross-analysis, with operators switching between both modalities, i.e., conventional endoscopic instruments and the Anubiscope. Because of the surgeon's lack of experience with conventional ESDs (i.e., only three completed gastric ESDs during a hands-on interventional endoscopy course organized at our institute in a porcine model), and because of the endoscopist's lack of training with the Anubiscope, resulting in an inability to complete an ESD in an explanted stomach, it was decided not to switch operators at this feasibility stage.

Irrespective of the groups, major ESD steps included the following: (1) scoring the area to be dissected with electrocautery marks using a needle knife (Boston Scientific, Natick, MA, USA); (2) submucosal injection of a fluid mixture of $10 \%$ glycerol and indigo carmine to create a cushion in the submucosal layer; and (3) circumferential precut mucosal incision using the insulated tip (IT) knife (Olympus KD-610L, Tokyo, Japan).

Anubiscope group

All procedures were performed by a laparoscopic surgeon with $\sim 30 \mathrm{~h}$ ' experience with the Anubiscope in procedures such as gastric ESDs in both pig and cadaver models, cholecystectomies in the pig model, and transrectal retroperitoneoscopic NOTES procedures [12] assisted by an endoscopist. The submucosal injection was performed by inserting the needle through the central 3.2-mm channel. Through the same channel, the IT knife was introduced to perform the mucosal precut incision. Submucosal dissection was performed with an endoscopic grasper and ad hoc electrocautery introduced through the two 4.2-mm working channels, manipulated with gun-shaped handles. When required, instruments could be switched between the left and the right hand to allow for the most convenient exposure, traction, and dissection.

\section{Conventional endoscopic group}

All procedures were performed by an experienced endoscopist with more than 300 ESDs performed in the clinical setting with the assistance of an operator who manipulated ancillary endoscopic instruments. A double-channel endoscope (13806 PKS; Karl Storz, Tüttlingen, Germany) with a transparent cap (D-201-13404, Olympus, Tokyo, Japan) as well as the ForceTriad (Covidien, Boulder, CO, USA) electrosurgical system were used. Submucosal dissection was performed with the IT knife.

At the end of the dissection, specimens were extracted and fixed on a plate of expanded polystyrene with pins. After the endoscopic procedure, a laparotomy was performed (Fig. 2). The colon and surrounding structures were inspected to exclude perforation or injury to adjacent organs. The colon was then resected and opened lengthwise, and resection sites were inspected from the inner and outer sites to ensure that all marked lesions were completely resected and to exclude perforation. Specimens were examined for size and resection margins.
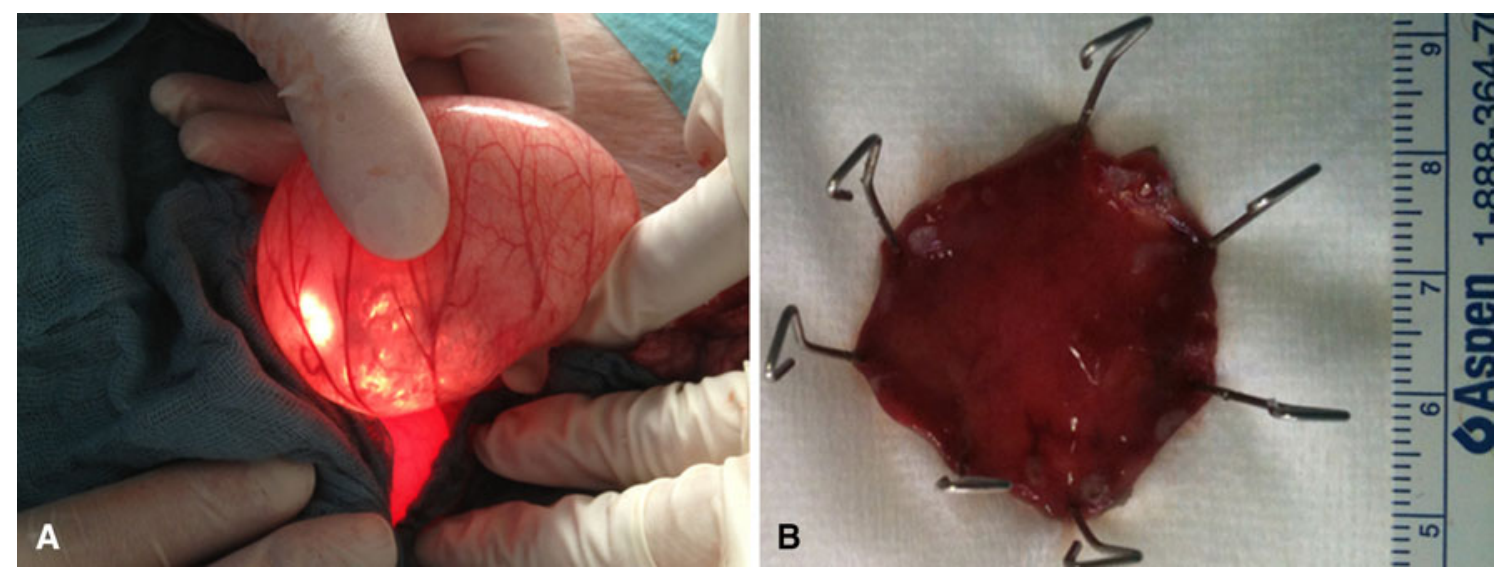

Fig. 2 Control laparotomy and ESD specimen. A Control laparotomy showing absence of perforation at the ESD site. B Colonic ESD specimen 


\section{Outcomes}

The following variables were recorded as follows: size of the resected specimen, total operative time, dissection time, dissection speed, achievement of en bloc resection, achievement of complete resection, and complications including perforation or bleeding.

En bloc resection was defined as resection in one piece. Complete dissection was defined if all scoring marks were included in the resected specimen. Total operative time was defined as the time required from marking the pseudotumors until complete removal of the lesion. Dissection time was defined as the time required only for submucosal dissection after circumferential cutting. Dissection speed was defined as the ratio between the dissected specimen surface and dissection time.

\section{Statistical analysis}

Statistics and graphs were performed by GraphPad Prism software, version 5. A $t$ test was used to calculate $p$ values in continuous variables. Fisher's exact test was used to calculate $p$ values in categorical variables.

\section{Results}

Nine ESDs were performed in three swine at 25, 15, and $10 \mathrm{~cm}$ above the anal verge with the Anubiscope. To obtain comparative data for porcine colonic ESDs, 16 ESDs were performed in five pigs with conventional endoscopic instruments.

The endoluminal surgical triangulation provided by the Anubiscope with the ability to expose and apply the required traction coupled with fine micromovements of operative instruments allowed for a smooth ESD (Video Clip 1 and Video Clip 2). The Anubiscope offers a panoramic view of the dissection field with increased visualization of the submucosal layer as opposed to the tubular view offered by the conventional endoscope (Video Clip 3 ), in which dissection is achieved advancing the scope's head underneath the mucosal layer and using in-line endoknives.

No perforations occurred in the Anubiscope group, while there were eight perforations $(50 \%)$ in the conventional group $(p=0.02)$. Complete and en bloc dissections were achieved in all cases in the Anubis group.

Mean dissection time for completed cases was statistically significantly shorter in the Anubis group (32.3 \pm 16.1 vs. $55.87 \pm 7.66 \mathrm{~min} ; p=0.0019)$.

Mean specimen size was larger in the conventional group $\left(1321 \pm 230\right.$ vs. $\left.927.77 \pm 229.96 \mathrm{~mm}^{2} ; p=0.003\right)$. Mean dissection speed (surface/time) was similar (35.95 \pm 18.93 vs. $23.98 \pm 5.02 \mathrm{~mm}^{2} / \mathrm{min}$ in the Anubis and conventional groups, respectively; $p=0.1$ ).

\section{Discussion}

ESD may achieve a complete en bloc resection of endoscopically treatable gastrointestinal lesions, irrespective of size.

Indication criteria for colorectal ESDs include laterally spreading tumors of nongranular type, with noninvasive patterns at magnification colonoscopy, which are larger

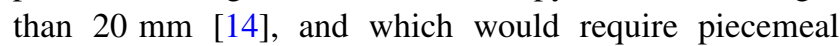
resection if treated by snare EMR [15].

ESDs are increasingly popular in Eastern countries, where aggressive mass screening programs, performed as a result of the higher incidence of gastrointestinal neoplasms, provided fundamentals for tailored minimally invasive approaches for early stage cancers [16]. Large disparities in outcomes, favoring Eastern patients, have also been reported.

In addition to epidemiology, limiting factors to the widespread adoption of ESDs in Western countries include technical challenges inherent to the procedure. A particularly delicate aspect is the lack of retraction on the specimen to be dissected, resulting in difficult exposure and a high risk of perforation.

To increase exposure and traction on the mucosal layer, Imaeda et al. proposed the use of an external grasping forceps held by a second forceps introduced into the working channel. The external forceps is used to grasp the lesion's margins and to expose the submucosal layer by oral traction [17].

Parra-Blanco et al. [3] have proposed custom-made internal retraction systems combining endoscopic clips applied to the resected mucosa's inner margin, attached through a rubber band to a second clip, which is placed on the normal distal mucosa, thus allowing self-standing traction and dissection plane visualization.

Similarly, Sakamoto et al. [7] introduced a spring-action clip, which offers adequate static tension of the specimen to be dissected. An ingenious solution, the pulley technique, offering internal triangulation and effective traction, has also been put forward [18, 19]. The technique implies placement of sutures functioning as fulcrum points on a gastric fold opposite to the ESD site and then anchored on the margins of the precut mucosa. The lift-up is achieved by pulling on the sutures coming out at the oral side. However, the pulley technique requires extra time to place fulcrum sutures and/or specific endoscopic suturing platforms.

The advantage of the surgical endoscopic platform used in the present study - the Anubiscope-lies in the fact that 


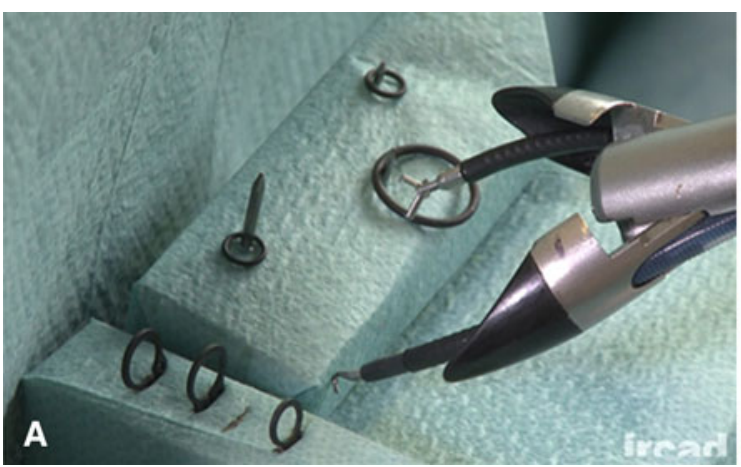

Fig. 3 Robotic version of the ISIS-SCOPE. A Master side. The operator is seated at the workstation and uses ergonomic handles with an intuitive motion pattern to command effectors reproducing human

it transfers surgical triangulation advantages, the mainstay of minimally invasive laparoscopic surgery, to the endoluminal environment. The Anubiscope allows for a controllable exposure of the submucosal layer and adequate traction to ensure precise dissection with minimum use of cautery and micromovements of working instruments.

The ergonomics of the Anubiscope is another strong asset of the device, with the possibility of manipulating manipulate working instruments (grasper and electrocautery) with both hands through two intuitive handles.

Colonic ESDs in the porcine model with the Anubiscope were significantly faster and more accurate, with a $100 \%$ completion rate and no perforations. In the conventional group, in the hands of an experienced endoscopist, the perforation rate was $50 \%$. It has to be emphasized that the porcine model is not appropriate for colonic ESDs because the mucosal layer is very thin, and the pigs used in this experimental study were quite small $(35-40 \mathrm{~kg})$.

The main drawback of this study's design lies in the lack of crossing between operators; both used their preferred approach. The original design was not respected because metrics obtained by operators with switched instruments from ex vivo training warranted longer training before a randomized trial could be ethically performed in animals. The main end point was to provide proof of the concept with regard to the effectiveness of the surgical endoscopic platform to perform ESDs, but a control group with conventional tools, in similar experimental conditions, although with different operators, was required to obtain comparative data.

The next sensible step is the creation of a program including training and innovation in hybrid laparoendoscopic techniques for both surgeons and endoscopists to acquire the necessary skills for safe and effective minimally invasive treatment of gastrointestinal lesions.

For this purpose, the IRCAD organizes flexible surgery courses, endorsed by the Society of American

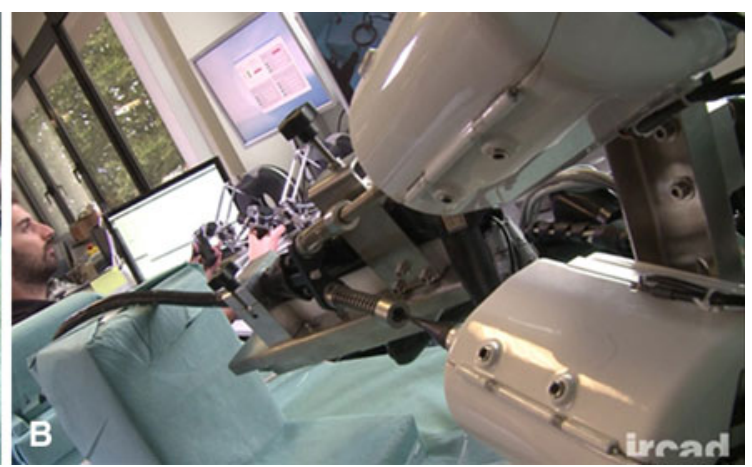

hand movements into a precise and downscaled fashion. B Slave side. Motors transfer the motion to the distal effectors

Gastrointestinal and Endoscopic Surgeons (SAGES) and by the European Association of Endoscopic Surgeons (EAES).

Additionally, in 2011, the IRCAD founded the University Hospital Institute (IHU) for Minimally Invasive Hybrid Image-guided Surgery, which is a newly created scientific foundation. Its mission is to create and develop an innovative hybrid surgery integrating the best minimally invasive and image-guided techniques for the treatment of abdominal diseases.

The goal is to create the next generation of hybrid physicians combining the best aspects of minimally invasive techniques from laparoscopic surgery, flexible endoscopy, and interventional radiology to create optimal hybrid approaches and achieve optimum patient care.

Furthermore, a robotic version of the Anubiscope, with an intuitive haptic interface allowing for smooth and controlled micromovements (Fig. 3), has been developed at the IRCAD within the ISIS project, which was funded by regional funding (region of Alsace, France) and by the European fund for Regional Development (FEDER). So far, it has been used to perform basic dry laboratory tasks, with unparalleled agility and the ability to transfer a sufficient amount of force for traction, suturing, and knot tying. Further developments are required to implement cautery and additional features for more complex surgical tasks such as ESDs.

\section{Conclusions}

Colonic ESDs in a porcine model proved to be feasible with the Anubiscope. This surgical endoscopic platform is promising for endoluminal surgical procedures such as ESD, as it is user-friendly, effective, and safe. A robot version of the Anubiscope is currently under development. 
Acknowledgments IRCAD-IHU Institutes are recipients of grants from Karl Storz Endoscopes, Tüttlingen, Germany; Covidien, Mansfield, MA, USA; and Siemens AG Healthcare, Munich, Germany. The authors acknowledge the European Funding for Regional Development (FEDER) and the Region of Alsace for supporting the ISIS project. The authors are grateful to Guy Temporal, Camille Fischer, and Christopher Burel for their valuable assistance.

Disclosures Michele Diana, Hyunsoo Chung, Keng-Hao Liu, Bernard Dallemagne, Nicolas Demartines, Didier Mutter, and Jacques Marescaux have no conflicts of interest or financial ties to disclose.

\section{References}

1. Saito Y, Fukuzawa M, Matsuda T, Fukunaga S, Sakamoto T, Uraoka T, Nakajima T, Ikehara H, Fu KI, Itoi T, Fujii T (2010) Clinical outcome of endoscopic submucosal dissection versus endoscopic mucosal resection of large colorectal tumors as determined by curative resection. Surg Endosc 24:343-352

2. Fujishiro M (2008) Perspective on the practical indications of endoscopic submucosal dissection of gastrointestinal neoplasms. World J Gastroenterol 14:4289-4295

3. Parra-Blanco A, Nicolas D, Arnau MR, Gimeno-Garcia AZ, Rodrigo L, Quintero E (2011) Gastric endoscopic submucosal dissection assisted by a new traction method: the clip-band technique. A feasibility study in a porcine model (with video). Gastrointest Endosc 74:1137-1141

4. Tanaka S, Oka S, Chayama K (2008) Colorectal endoscopic submucosal dissection: present status and future perspective, including its differentiation from endoscopic mucosal resection. J Gastroenterol 43:641-651

5. Saito Y, Uraoka T, Matsuda T, Emura F, Ikehara H, Mashimo Y, Kikuchi T, Fu KI, Sano Y, Saito D (2007) Endoscopic treatment of large superficial colorectal tumors: a case series of 200 endoscopic submucosal dissections (with video). Gastrointest Endosc 66:966-973

6. Fujishiro M, Yahagi N, Kakushima N, Kodashima S, Muraki Y, Ono S, Yamamichi N, Tateishi A, Oka M, Ogura K, Kawabe T, Ichinose M, Omata M (2007) Outcomes of endoscopic submucosal dissection for colorectal epithelial neoplasms in 200 consecutive cases. Clin Gastroenterol Hepatol 5:678-683

7. Sakamoto N, Osada T, Shibuya T, Beppu K, Matsumoto K, Mori H, Kawabe M, Nagahara A, Otaka M, Ogihara T, Watanabe S (2009) Endoscopic submucosal dissection of large colorectal tumors by using a novel spring-action S-O clip for traction (with video). Gastrointest Endosc 69:1370-1374

8. Neuhaus H, Costamagna G, Deviere J, Fockens P, Ponchon T, Rosch T (2006) Endoscopic submucosal dissection (ESD) of early neoplastic gastric lesions using a new double-channel endoscope (the "R-scope"). Endoscopy 38:1016-1023

9. Yonezawa J, Kaise M, Sumiyama K, Goda K, Arakawa H, Tajiri $H$ (2006) A novel double-channel therapeutic endoscope ("Rscope") facilitates endoscopic submucosal dissection of superficial gastric neoplasms. Endoscopy 38:1011-1015

10. Dallemagne B, Marescaux J (2010) The ANUBIS ${ }^{\mathrm{TM}}$ project. Minim Invasive Ther Allied Technol 19:257-261

11. Dhumane PW, Diana M, Leroy J, Marescaux J (2011) Minimally invasive single-site surgery for the digestive system: a technological review. J Minim Access Surg 7:40-51

12. Leroy J, Diana M, Barry B, Mutter D, Melani AG, Wu HS, Marescaux J (2012) Perirectal Oncologic Gateway to Retroperitoneal Endoscopic Single-Site Surgery (PROGRESSS): a feasibility study for a new NOTES approach in a swine model. Surg Innov 19:345-352

13. Perretta S, Dallemagne B, Barry B, Marescaux J (2013) The ANUBISCOPE $^{\circledR}$ flexible platform ready for prime time: description of the first clinical case. Surg Endosc 27(7):2630

14. Uraoka T, Saito Y, Matsuda T, Ikehara H, Gotoda T, Saito D, Fujii T (2006) Endoscopic indications for endoscopic mucosal resection of laterally spreading tumours in the colorectum. Gut 55:1592-1597

15. Shono T, Ishikawa K, Ochiai $Y$, Nakao M, Togawa O, Nishimura M, Arai S, Nonaka K, Sasaki Y, Kita H (2011) Feasibility of endoscopic submucosal dissection: a new technique for en bloc resection of a large superficial tumor in the colon and rectum. Int J Surg Oncol 2011:948293

16. Bickenbach K, Strong VE (2012) Comparisons of gastric cancer treatments: East vs. West. J Gastric Cancer 12:55-62

17. Imaeda $H$, Iwao $Y$, Ogata $H$, Ichikawa $H$, Mori M, Hosoe $N$, Masaoka T, Nakashita M, Suzuki H, Inoue N, Aiura K, Nagata H, Kumai K, Hibi T (2006) A new technique for endoscopic submucosal dissection for early gastric cancer using an external grasping forceps. Endoscopy 38:1007-1010

18. Rieder E, Makris KI, Martinec DV, Swanstrom LL (2011) The suture-pulley method for endolumenal triangulation in endoscopic submucosal dissection. Endoscopy 43(suppl 2 UCTN):E319-E320

19. Li CH, Chen PJ, Chu HC, Huang TY, Shih YL, Chang WK, Hsieh TY (2011) Endoscopic submucosal dissection with the pulley method for early-stage gastric cancer (with video). Gastrointest Endosc 73:163-167 